\title{
Article \\ Towards a Physically Consistent Phase-Field Model for Alloy Solidification
}

\author{
Peter C. Bollada ${ }^{1, *, \dagger}$, Peter K. Jimack ${ }^{2, \dagger}$ and Andrew M. Mullis ${ }^{1, \dagger}$ \\ 1 School of Chemical and Process Engineering, University of Leeds, Leeds LS2 9JT, UK; a.m.mullis@leeds.ac.uk \\ 2 School of Computing, University of Leeds, Leeds LS2 9JT, UK; p.k.jimack@leeds.ac.uk \\ * Correspondence: p.c.bollada@leeds.ac.uk \\ + These authors contributed equally to this work.
}

check for

updates

Citation: Bollada, P.C.; Jimack, P.K.; Mullis, A.M. Towards a Physically Consistent Phase-Field Model for Alloy Solidification. Metals 2022, 12, 272. https://doi.org/10.3390/ met12020272

Academic Editor: Zhongyun Fan

Received: 16 December 2021

Accepted: 30 January 2022

Published: 2 February 2022

Publisher's Note: MDPI stays neutral with regard to jurisdictional claims in published maps and institutional affiliations.

Copyright: () 2022 by the authors Licensee MDPI, Basel, Switzerland. This article is an open access article distributed under the terms and conditions of the Creative Commons Attribution (CC BY) license (https:// creativecommons.org/licenses/by/ $4.0 /)$.

\begin{abstract}
We give an overview of contributions made to the computational phase-field modelling of alloy solidification from the University of Leeds as part of the LiME project (EPSRC Advanced Manufacturing Hub in Liquid Metal Engineering). The broader look the more salient features from our research allows the individual contributions to be seen in a wider context than can be seen from each contribution separately. We begin with a general introduction to phase-field and then reference the numerical issues that arise from the solution of the model before outlining contributions to phase-field modelling that we found most interesting or significant. These range from controlling and developing interface-width independent modelling; controlling morphology in both single and multiphase settings; generalising from single to multiphase models; and creating a thermodynamically consistent framework for modelling entropy flow and thereby postulating a temperature field consistent with the concepts of, and applicable in, multiphase and density-dependent settings.
\end{abstract}

Keywords: phase-field; alloy solidification; non-equilibrium thermodynamics; intermetallics

\section{An Introduction to Phase-Field Modelling}

The process of solidification of metals and alloys can be modelled using non-equilibrium thermodynamics. The principle difference between non-equilibrium and equilibrium thermodynamics is the existence of fields, such as temperature or concentration, that change in time. Phase-field modelling adds another principle field, so designed to provide a parameter that changes rapidly but continuously between the phases of matter of interest.

To familiarise the reader with key variable notation, we note that for pure metal solidification, the principle field is the temperature field, $T$, but for alloy solidification, the principle field is the conserved solute concentration field, $c$, and $T$ often plays a less important role-see, for examples, [1-3]. The phase-field symbol, $\phi$, and its multiphase relations, $\phi_{i}, i \in[1, n]$ when $n>2$, is an example of an order parameter, which typically takes the value of one in each of the phases of matter. For example, for two phases, $\phi_{1}=1$ may represent the liquid phase and $\phi_{2}=1$ the solid phase. Since the material must be one or the other, we have the constraint, $\phi_{1}+\phi_{2}=1$, and it becomes more convenient to use the single symbol, $\phi$, with its extreme values representing two phases, e.g., $\phi=1$ liquid, and $\phi=0$ the solid. However, in general, there can be more than one solid phase necessitating a multiphase formulation (in which case $\sum_{i=1}^{n} \phi_{i}=1$ ). It is often stated that the role of $\phi$ is to track the surface of solid growth. Though this is one of its roles, its other major role is to express the free energy associated with the surface. For a more detailed review of the role of phase-field modelling in solidification, the reader may consult [4-6], and the issues surrounding multiphase modelling [7].

A distinction brought into sharp focus in the work of [8] is that of the division of all classical physical phenomena into conserved and non-conserved (or dissipative) energy systems. The principle behind much of the phase-field modelling is the principle of optimal free energy minimisation, which firmly establishes phase-field solidification as modelling 
dissipative phenomena. The word "optimal" is crucial, since without it the principle is ill defined. The main objective of phase-field modelling of solidification is to provide insight into the role of free energy minimisation in the resulting morphology of solidifying melts. The modelling consists of three fundamental features: (1) Formation of the free energy in terms of the variables; (2) specification of the diffusion parameters of the system; (3) formation of the equations of motion by gradient descent of the free energy. A useful analogy for this process is that of descending down a hillside by, at each instant, choosing a path of steepest gradient and moving at a speed proportionate to the gradient. For the hill side analogy, the two-dimensional equations would be

$$
\dot{\mathbf{x}}=-M \nabla h
$$

or, in component form,

$$
\dot{x}_{i}=-M \frac{\partial h}{\partial x_{i}}, i=1,2 .
$$

Here, $x_{1}, x_{2}$ are the two Cartesian directions in space (more conventionally denoted $x, y$ and ( $z$ for 3D), which we will use interchangeably with $x_{i}$ where convenient); $\dot{\mathbf{x}}$ is the velocity; $h$ is the height in coordinates $x_{1}, x_{2}$; and the parameter, $M>0$, which may be a function of $x_{1}$ and $x_{2}$, controls the response to the driving gradient $\nabla h$. Furthermore, notice the negative sign that enforces reduction rather than an increase in height. Such a descent is illustrated in Figure 1 following the dashed line, which is always perpendicular to contours of equal height.

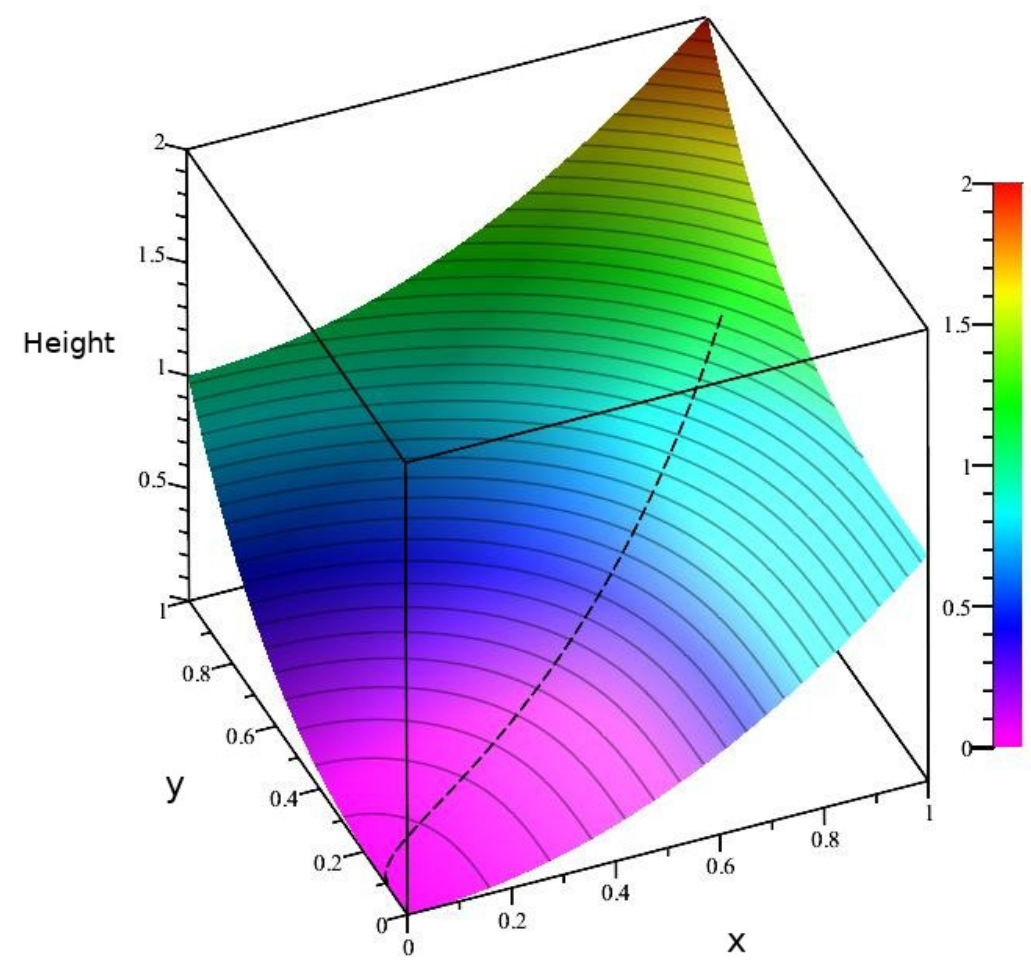

Figure 1. A path down a hill side following the path of steepest descent. The descending particle moves more quickly when the slope is steep (towards the top in this case) but slows down as it approaches a local minimum (near the bottom in this case). 
The phase equation for the evolution of $\phi$ is very similar in the form:

$$
\dot{\phi}=-M \frac{\delta F}{\delta \phi}
$$

where $M$ controls the speed of descent-the mobility. $F$ is the free energy of the domain as a whole. In 3D this is

$$
F=\int f(\phi, c) \mathrm{d} x_{1} \mathrm{~d} x_{2} \mathrm{~d} x_{3}
$$

where the free energy density, $f$, is given in general by gradients and functions of $\phi$ and $c$ (and also $T$ if necessary). Variational techniques (using the notation discussed in [8]) convert the variational notation into functions of phase, $\phi$, and its gradients, $\nabla \phi$ :

$$
\frac{\delta F}{\delta \phi}=-\nabla \cdot \frac{\partial f}{\partial \nabla \phi}+\frac{\partial f}{\partial \phi} .
$$

In Equation (1), the path has two degrees of freedom. On the other hand, since the function, $\phi(\mathbf{x}, t)$, is defined by all its values throughout the 2D (or 3D) domain, Equation (3) has infinite degrees of freedom. To understand this consider $\phi$ to be approximated by its value at $N$ points, $\Phi_{i}$, for $i=1 \ldots N$. Then this system would involve $N$ degrees of freedom, with equations

$$
\dot{\Phi}_{i}=-M \frac{\partial F}{\partial \Phi_{i}}, i=1 \ldots N
$$

where the similarity with the component form of hill descent, Equation (2), is clear.

In this way, the specification of $F$, being the integral of the density, $f$, across the whole domain leads towards a field equation, which gives the evolution of $\phi$ at every point. By design, one feature of the phase is that it is not a conserved quantity in solidification. Non-conservation of phase is essential to allow the liquid phase to be replaced by the solid phase as solidification progresses. For the solute concentration, a similar equation to Equation (5) would lead to the undesirable consequence of an increase in one of the components of the binary alloy. To that end, a Cahn-Hilliard equation in the form

$$
\dot{c}=\nabla \cdot D \frac{\partial f}{\partial c}
$$

is used for solute evolution (typically, gradients of solute, $c$, are not used in the formation of free energy). Here $D$ is the solute diffusion, which is always (for substitutional diffusion) several orders smaller in the solid than in the liquid, and written (in the two phase case) $D=\phi D_{\text {Liquid }}+(1-\phi) D_{\text {Solid }}$, where $D_{\text {Liquid }} \gg D_{\text {Solid }}$.

\section{Numerical Techniques}

Once the free energy density is specified, the evolution equations are known given the initial conditions. For binary alloy models, the phase-field initial condition consists of an initial nucleus, without which the system would remain in a permanent metastable state of undercooled liquid. This nucleus must be the solid phase (not just a surface such as a boundary or another phase). The initial condition for solute consists of a boundary value and often a value that differs in the solid region.

The evolution equations can rarely be solved analytically, even in 1D. Considerable computational resources are frequently required alongside state-of-the-art numerical techniques. Two factors make the solution problematic: one, the equations are nonlinear; two, there is much activity on the growing surface, but the field throughout a large proportion of the domain is also required. If both solute and temperature equations are added to the phase equations, realistic diffusion parameters stretch the size of the domain to orders of 
magnitude larger than the solid region. The surface itself is often fractal in appearance, so even if a locally adaptive grid is used, the degrees of freedom can be prohibitive, see [9] for a detailed discussion of numerical techniques brought to bear on the model of [3].

In [10], we introduce a numerical framework and associated software that combines multiple state-of-the-art techniques to provide an efficient and reliable approach to the computational simulation of phase-field systems for binary alloy solidification. Specifically, Reference [10] is the first tool that successfully combines each of the following components: fully implicit time integration; adaptive mesh refinement and coarsening in three dimensions; use of nonlinear multigrid iterations; and the scalable use of parallel processing. The implicit time discretisation is ideally suited to the stiff systems of nonlinear ordinary differential equations that arise following spatial discretisation. This stiffness is a result of the very different (by multiple orders of magnitude) rates of diffusion in the temperature and the solute equations, and results in excessively small time-steps being required for most numerical integration schemes. We are able to overcome this in [10] by using fullyimplicit time-stepping, based upon the BDF (backwards differentiation formulae) family. The consequence, however, is that a very large system of nonlinear algebraic equations must be solved at each time step. The use of mesh adaptivity allows the spatial discretisation to focus on the regions of the computational domain that are of most significance at any point in time (i.e., the current location of the phase interfaces and the regions just ahead of them) and, therefore, reduces the size of this nonlinear system very substantially without losing accuracy in the simulation. In order to implement this adaptivity, we use a hierarchy of meshes, which has the dual purpose of allowing coarsening once the phase interface has passed through a region and of supporting the use of multigrid methods to solve the nonlinear algebraic system of equations at each time step. In [10], we use a nonlinear multigrid algorithm called FAS (full approximation scheme) and are able to obtain solutions in a computational time that is almost linear with respect to the number of degrees of freedom in the spatial grid. Finally, we implement all of the above within a parallel computing framework to obtain further speed-ups in the overall solution time.

Subsequent to the publication of [10], we have continued to make further improvements to the software. For example, in order to implement the multigrid solver, it is necessary to compute derivatives of the nonlinear residual at each time step with respect to each of the degrees of freedom in the spatial discretisation. This can be performed analytically (as in [10]); however, these derivatives may also be approximated numerically. This has the dual advantage of being computationally faster and (perhaps more importantly) of not requiring changes to this section of the software when the physical system is changed (e.g., for the simulation of different alloys, requiring alterations to the phase-field equations). Another, more recent, development is to improve the efficiency of the parallel implementation, as discussed in [11]. When parallelism is obtained through geometrically partitioning the simulation across the available processes, it is necessary to provide an equal number of degrees of freedom to each of the processors in order to maximize efficiency. When adaptivity occurs, the computational grid changes, with some processors now having more degrees of freedom and others fewer-this creates a work imbalance that needs to be corrected (this is known as dynamic load-balancing). Historically, dynamic load-balancing algorithms have only taken account of the number of degrees of freedom in the computational grid that are assigned to each processor: in [11], we showed that, when applying a multigrid solver, it is advantageous to also consider the way in which the entire hierarchy of meshes is partitioned across the processing units. This results in improved utilisation of the parallel processing resources and faster computational times (allowing more accurate or longer simulations for a given compute budget).

Note that the contributions of $[10,11]$, whilst using phase-field simulation as the motivation, are primarily around our development and understanding of advanced numerical methods. Consequently, these advances can also be applied to much wider classes of nonlinear time-dependent problems, as demonstrated in [12]. 


\section{Free Energy Formation}

For simplicity of exposition, we focus now on systems with only two phases present. Multiphase will be revisited briefly in Section 6 and subsequently in Section 8.

Formation of the free energy density, $f$, is where much of the modelling takes place. The free energy density is a sum of two parts: the surface, $f_{S}$, and the bulk, $f_{B}$ :

$$
f=f_{S}(\nabla \phi, \phi, c)+f_{B}(\phi, c)
$$

For the simplest binary alloy model, $f_{S}$ is given by

$$
f_{S}=\frac{1}{2} \delta^{2} \nabla \phi \cdot \nabla \phi+\frac{1}{2} \phi^{2}(1-\phi)^{2}
$$

where $\delta$ has the units of length and is associated with the thickness of the transition region between liquid and solid (here and throughout this article we use a dimensionless form for energy for simplicity of expression-see Appendix A). This model gives isotropic growth. The first term (containing the gradient) gives a penalty for a gradient in phase, which, if acting alone, would destroy the initial condition and melt the alloy. The other term known as the double-well potential, $\Omega \equiv \frac{1}{2} \phi^{2}(1-\phi)^{2}$ (both nongradient and nonlinear), has the opposite effect and, acting alone, would intensify the gradient towards infinity. The two terms acting together give a reasonably constant interface thickness with a transition well approximated by a sigmoid (see Equation (13)).

\section{Phase Profile}

Considering just the surface contribution in $1 \mathrm{D}$ so that

$$
F_{S}=\int f_{S} \mathrm{~d} x=\int\left[\frac{1}{2} \delta^{2} \frac{\partial \phi^{2}}{\partial x}+\frac{1}{2} \phi^{2}(1-\phi)^{2}\right] \mathrm{d} x
$$

then setting the variational derivative of this to zero,

$$
\frac{\delta F_{S}}{\delta \phi}=0
$$

implies the differential equation:

$$
\delta^{2} \frac{\partial^{2} \phi}{\partial x^{2}}=\frac{\partial}{\partial \phi}\left[\frac{1}{2} \phi^{2}(1-\phi)^{2}\right]=3 \phi^{2}-4 \phi+1 .
$$

Equation (12) is solved by the phase profile

$$
\phi=\frac{1}{1+\exp (-x / \delta)}
$$

which is near zero for $x \ll 0$ and near unity for $x \gg 0$ with a transition region in proportion to $\delta$ about $x=0$. The phase profile, Equation (13), thus satisfies just the surface contributions to the phase-field, Equation (12). This may prompt the question: How is the phase profile controlled in general, say, to match a specific profile? Equation (13) may be rearranged

$$
\frac{x}{\delta}=\ln (1-\phi)-\ln \phi
$$

By considering a generalisation of Equation (14) in the form of

$$
X \equiv \frac{x}{\delta}=a_{1} \ln (1-\phi)+a_{2} \ln \phi+a_{3}
$$


it becomes possible to use the coefficients $a_{1}, a_{2}$ and $a_{3}$ to fit a desired profile to data from, say, a molecular dynamics simulation or ab initio calculation (see, for example, [13]). As shown in [14], the form of the potential well, $\Omega$, necessary for a skewed profile is given by

$$
\Omega=\frac{1}{2}\left(\frac{\partial X}{\partial \phi}\right)^{-2}
$$

and the form for the interpolation polynomial, $g$, is given by

$$
g(\phi) \propto \int \sqrt{\Omega} \mathrm{d} \phi
$$

The reader can obtain a detailed discussion of the effect of interpolating functions in [15]. A derivation of the above Equations is given in [14], where an application of the method to real data is shown in Figure 2. Here, the transition in space from liquid (unstructured) to solid (structured) is over a distance $\delta$, normalised to unity. Note that it is more convenient to represent the space variable, $X$, as a function of the phase variable, $\phi$, so that Equations (15) and (16) can be applied directly. We observe that the near sigmoid shape of the molecular dynamic computed points (blue asterisk in the left of Figure 2) results in a near standard-looking double-well potential, approximately proportional to $\phi^{2}(1-\phi)^{2}$, but the ab initio data leads to a double-well skewed significantly towards the solid.
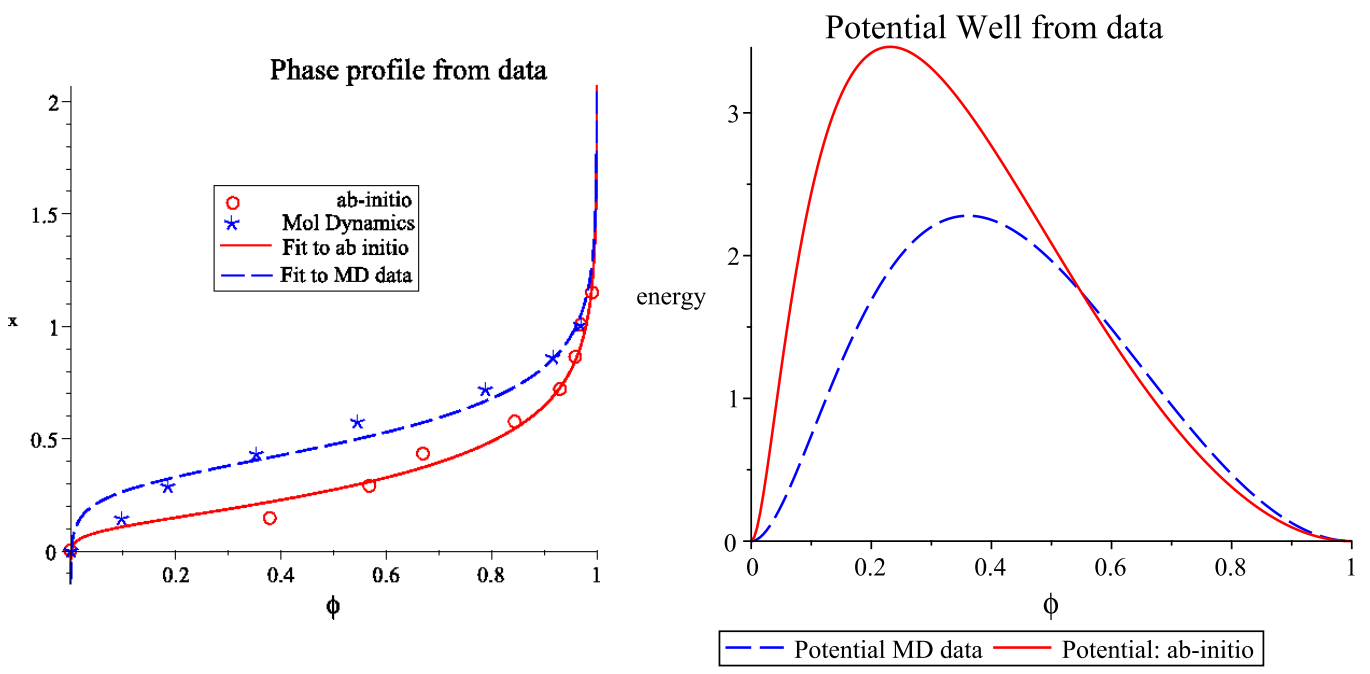

Figure 2. On the left figure, the blue asterisks represent 8 intervening transition points in space (normalised between $x=0$ and $x=1$ ) and computed using molecular dynamics. The points lie between liquid and solid with values of $\phi=1$ being completely crystalline and $\phi=0$ being completely unstructured. Similarly, red circles represent the same transition but from ab initio calculations. We fit two curves to the data, which result in the two corresponding potential wells in the right figure.

The significance of the paper lies in the application of phase-field modelling of crystal growth where physically real interface widths, and therefore shape, are of interest.

\section{Anisotropy}

Anisotropy is typically introduced into phase-field models by the addition of an anisotropy term, $A(\nabla \phi)$, in the following manner

$$
f_{S}=\frac{1}{2} \delta^{2} A^{2} \nabla \phi \cdot \nabla \phi+\frac{1}{2} \phi^{2}(1-\phi)^{2}
$$


The anisotropy, $A$, is dimensionless, but because of the presence of gradients in its definition, considerably adds to the nonlinearity. Referring to an earlier point about model dependent terms in Section 2, changing the anisotropy changes the linearisation in the numerical scheme. In the phase-field modelling literature, probably the most explored form of $A$ is that of the 2D four-fold anisotropy (not withstanding its limited use in nature, since $2 \mathrm{D}$ systems are rare):

$$
A=1+\epsilon \cos (4 \theta)
$$

where the new variable, $\theta$, is related to the phase variable

$$
\cos \theta=\frac{\phi_{x}}{\sqrt{\phi_{x}^{2}+\phi_{y}^{2}}}
$$

where $\phi_{x}, \phi_{y}$ is shorthand for the $x$ and $y$ derivative of $\phi$, respectively.

The effect of this example of $A$ is to give a small preferential growth direction along the Cartesian $x$ and $y$ axes. When the driving force is strong, this results in highly directional growth-dendrites (see Figure 3 illustrating 3D growth using an extension of Equation (19), shown below as

$$
\cos (4 \theta) \equiv 4\left(\cos ^{4} \theta+\sin ^{4} \theta\right)-3,
$$

implying that the 2D anisotropy can be written

$$
\begin{aligned}
A=1+\epsilon \cos (4 \theta) & \equiv 1+\epsilon\left[4\left(\cos ^{4} \theta+\sin ^{4} \theta\right)-3\right] \\
& =1+\epsilon\left[4 \frac{\phi_{x}^{4}+\phi_{y}^{4}}{\left(\phi_{x}^{2}+\phi_{y}^{2}\right)^{2}}-3\right] .
\end{aligned}
$$

This extends naturally to 3D anisotropy as

$$
A=1+\epsilon\left[4 \frac{\phi_{x}^{4}+\phi_{y}^{4}+\phi_{z}^{4}}{\left(\phi_{x}^{2}+\phi_{y}^{2}+\phi_{z}^{2}\right)^{2}}-3\right]
$$

Emergence of these highly complex morphologies continues to excite phase-field modellers to date. For example, it is possible with highly parallel computers to simulate a large number of dendrites with different preferential growth directions [16].

The role of the parameter, $\epsilon$, in the anisotropy is interesting. When $\epsilon=0$, we return isotropy. However, there is a value, $\epsilon=1 / 15$ in Equation (19), beyond which the resulting predicted equilibrium morphology in unstable. At the critical value, the function develops points of inflection (see Figure 4). Now, it is widely known (and our paper [17] discusses this ) that a point of inflection in the anisotropy is associated with a discontinuity in the associated equilibrium shape (also called the Wulff shape). The equilibrium shape, $W$, is given parametrically by

$$
W(t)=\left[x=A(t) \cos t-A^{\prime}(t) \sin t, y=A(t) \sin t+A^{\prime}(t) \cos t\right]
$$

and is illustrated with three values of $\epsilon$ in Figure 5 (note, these diagrams are theoretical, and for values of $\epsilon \geq 1 / 15$, must be interpreted with care). 


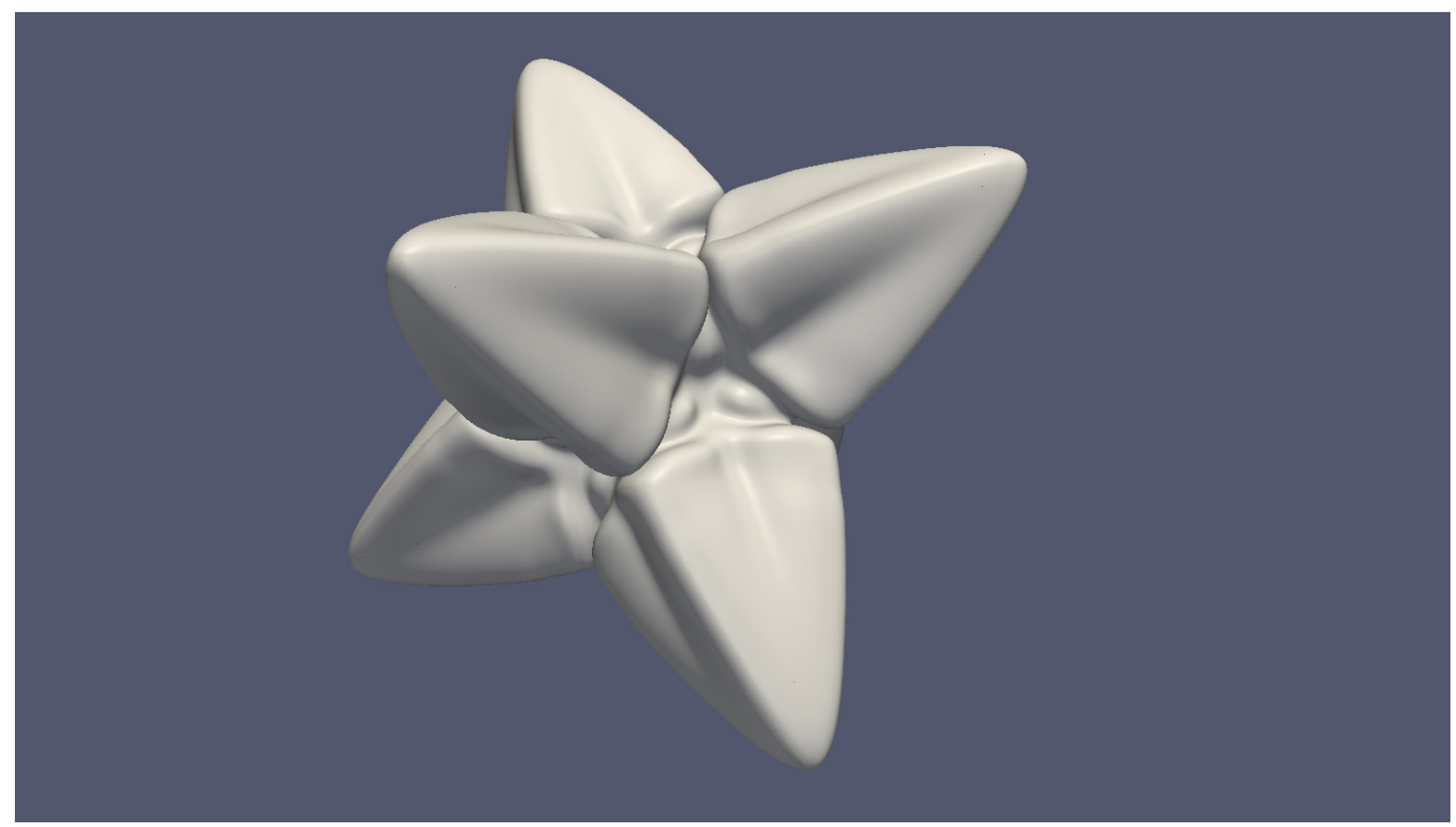

Figure 3. Dendrite growth with a 3D extension of the model anisotropy given in Equation (19).

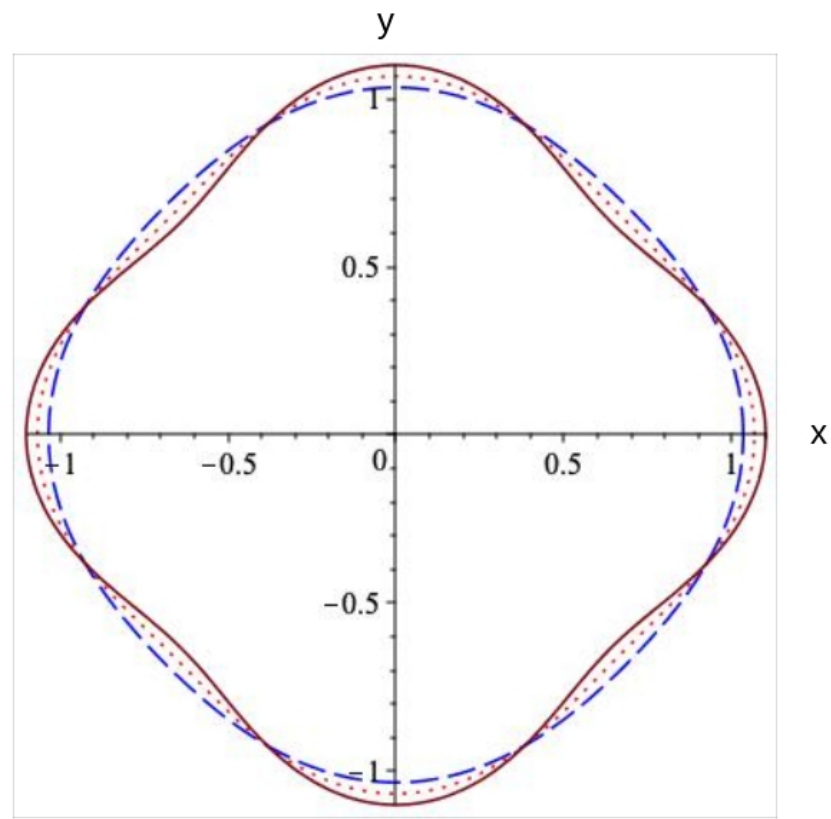

Figure 4. A polar plot of the anisotropy function Equation (19) for a range of values: $\epsilon=1 / 30,1 / 15,1 / 10$. These are, respectively: dashed blue; dotted red; and solid brown. For values of $\epsilon<1 / 15$, the polar plot has positive curvature, but for values of $\epsilon>1 / 15$, the plot develops points of inflection.

This has not prevented some researchers from using values of $\epsilon \gg 1 / 15$, with some stabilisation techniques in order to simulate faceted growth [18-20]. After first following standard literature to explore the growth of a regular hexagon in [21], the transition from pure faceted to dendritic morphology was investigated. Figure 6, reproduced from this paper shows the progressive effect of increasing undercooling from near equilibrium (regular polygon) to high undercooling (multifaceted-snowflake-like in appearance). The paper observes that, for higher undercooling, the transition for circular seed always passes through a polygon stage on the way to multifaceted. The paper thus addresses the question 
as to whether there is a cut-off undercooling for which at any smaller undercooling the polygon is the end state-no matter how long the simulation time. The answer argued is, yes, such a cut-off exists.

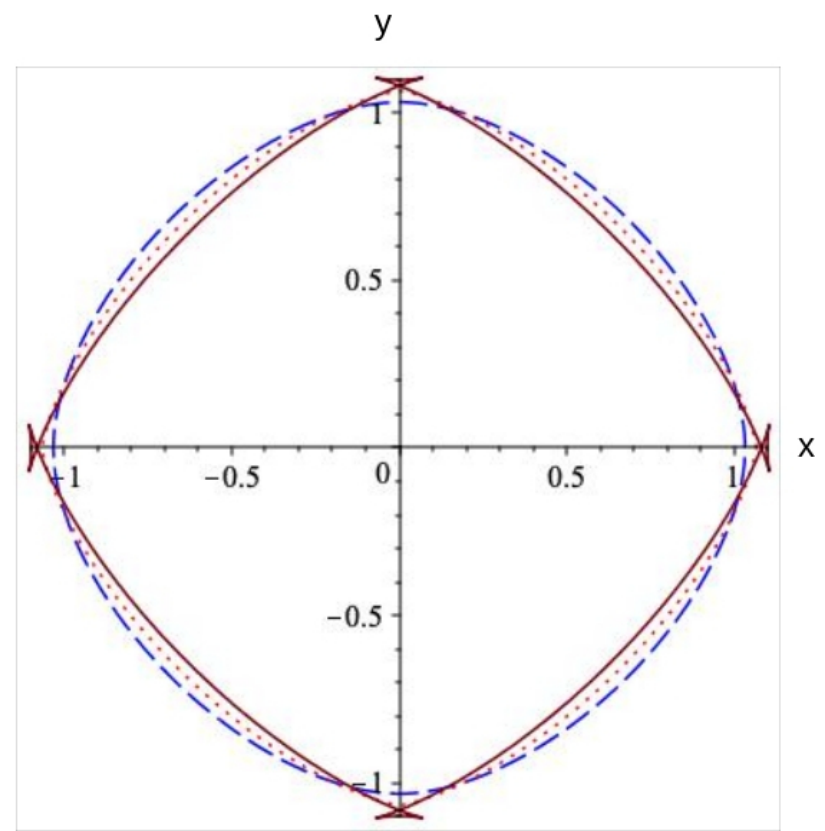

Figure 5. A Wulff plot of the anisotropy function Equation (19) for a range of values: $\epsilon=1 / 30,1 / 15,1 / 10$. These are, respectively: dashed blue; dotted red; solid brown. For values of $\epsilon<1 / 15$, the Wulff shape is continuous, but for values of $\epsilon>1 / 15 \mathrm{~m}$ the plot develops cusps (in the solid brown line).

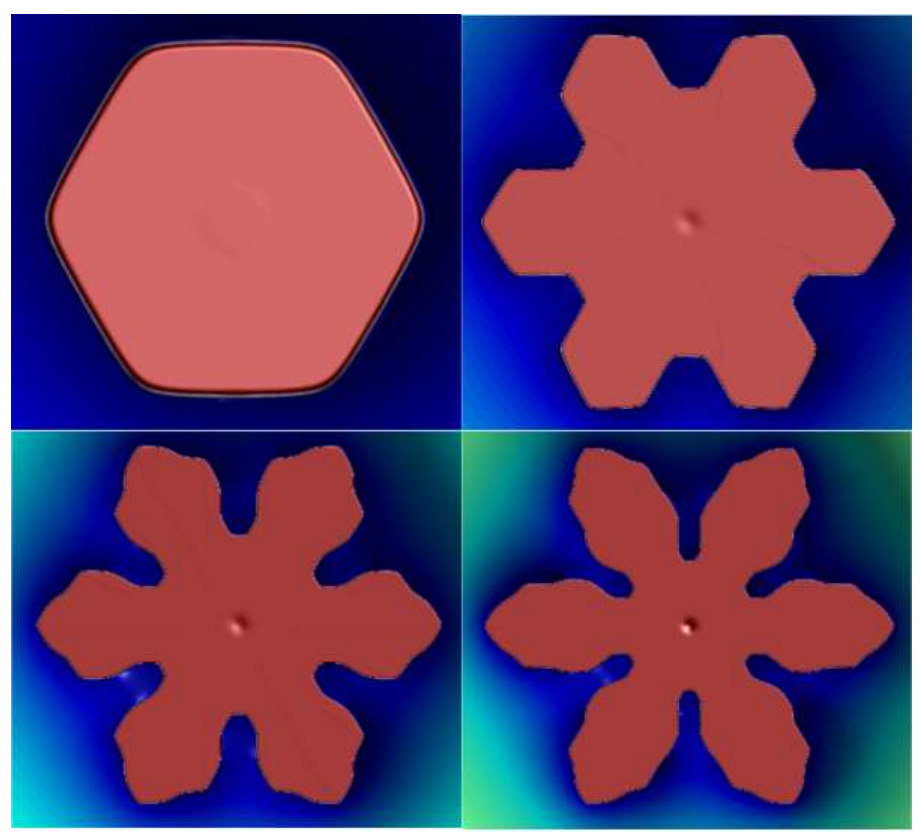

Figure 6. Clockwise from top left, the morphology for $T=1300 \mathrm{~K}, T=1200 \mathrm{~K}, T=1000 \mathrm{~K}$ and (bottom left) $T=1100 \mathrm{~K}$. Each plot is a function of solute concentration, the solid being effectively pure metal $(c=1)$. The blue area around the crystal indicates concentration depletion close to the growing edge $c \sim 0.3$. for $T=1000 \mathrm{~K}$. 
We argue, in our paper [17], that there is a cleaner, more flexible approach to facet modelling. To introduce faceting into the model, we first specify the vertices and give the following formula for anisotropy:

$$
A=\max \left(\frac{\nabla \phi}{|\nabla \phi|} \cdot \mathbf{p}_{i}, i=1 \ldots n\right)
$$

where $\mathbf{p}_{i}$ are the $n$ vertices of the resulting required equilibrium morphology. The quantity, $\mathbf{n} \equiv \nabla \phi /|\nabla \phi|$ is the normal to the surface, and so when the normal is in the same direction as one of the vertices, say, $\mathbf{p}_{3}$, then $A$ takes on the magnitude of $\mathbf{p}_{3}$. For $2 \mathrm{D}$ shapes, $A$ becomes a function of angle, $\theta$, and it is seen to be a series of connected circular arcs that pass through the vertices and meet on each facet. In $3 \mathrm{D}$, the surface energy is generalised to a series of connected spheres (see Figure 7), being an application of Equation (25) with the vertices of a cube, $\mathbf{p}_{i}, i=1 \ldots 8$ serving to define $A$.

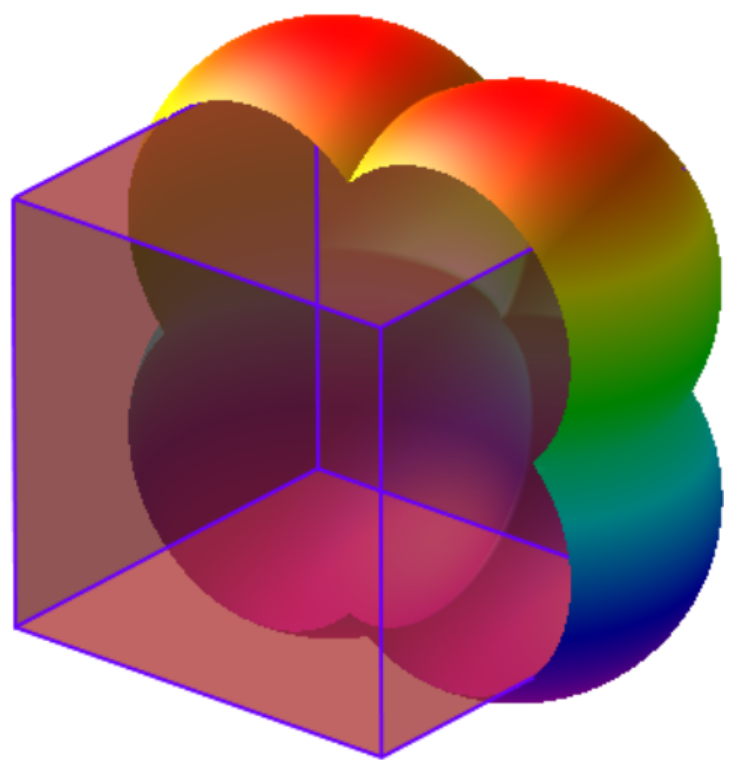

Figure 7. Anisotropy for a cube shown with half the anisotropy cut away. The cube vertices are at the points of maximum anisotropy and there is a cusp minimum at the centre of all faces.

The real advantage of Equation (25) is its flexibility to introduce any shape into the phase-field and then observe the growth towards the equilibrium shape (and beyond). For example, in Figure 8, we show the result of a phase-field simulation taken at two times, with the surface of a hexagonal prism (at the later time) displayed transparently so that the early growth can be seen to compare shape and size. Starting from a spherical seed (not shown), we see the flat faces form before the edges. 


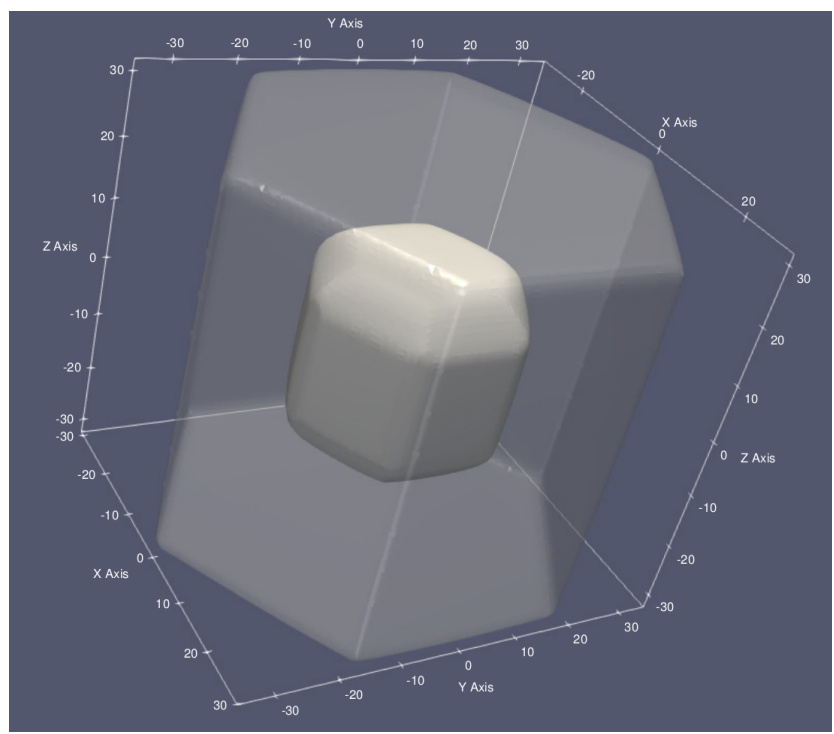

Figure 8. Mature hexagonal prism viewed in a semi-transparent perspective with an earlier growing crystal superimposed. The initial seed is a sphere of radius 10 and so the crystal growth forms the faceted face before the edges and vertices.

\section{Bulk Free Energy}

One of the more versatile forms of bulk free energy is that of an ideal binary solution, which consists of an entropy of mixing term plus a linear term for each phase (no RedlichKister terms):

$$
f_{i}(c)=c \ln c+(1-c) \ln (1-c)+\Delta_{i} c+a_{i}
$$

(see [22]) where $\Delta_{i}, a_{i}$ are given constants. A traditional method for combining these separate energies into a bulk free energy, $f_{B}$, is given by

$$
f_{B}=\sum_{i=1}^{n} g_{i} f_{i}(c)
$$

where, assuming a suitable interpolation function (i.e., monotonically increasing between $g(0)=0$ and $g(1)=1$, and with zero gradients at the extremes, $\left.g^{\prime}(0)=g^{\prime}(1)=0\right)$,

$$
g_{i}=\frac{g\left(\phi_{i}\right)}{\sum_{j=1}^{n} g\left(\phi_{j}\right)}
$$

prescribes a set of normalized functions $\left(\sum_{i} g_{i}=1\right)$, for multiphase interpolation.

For two phase, the formulation of the bulk free energy reads

$$
f=\frac{1}{2} A^{2} \delta^{2} \nabla \phi \cdot \nabla \phi+\frac{1}{2} \phi^{2}\left(1-\phi^{2}\right)+g(\phi) f_{\text {liq }}(c)+(1-g(\phi)) f_{\mathrm{sol}}(c)
$$

where $f_{\text {liq }}(c), f_{\text {sol }}(c)$ are the given bulk free energy densities for each phase of the binary alloy; possibly of the form given in Equation (26).

\section{Solute Trapping}

For many researchers, e.g., [23] the KKS method [24], and its theoretic relation [25], is the method of choice for constructing the bulk free energy for intermetallic alloys, as opposed to WBM [22]. We briefly illustrate the two methods in Figure 9. As should be evident from Figure 9, the method for interpolating the two pure phases using KKS is preferred for such free energy functions. On the other hand, for a solid solution, it can be 
more convenient to use WBM, which often allows the direct use of physically realistic free energy functions.
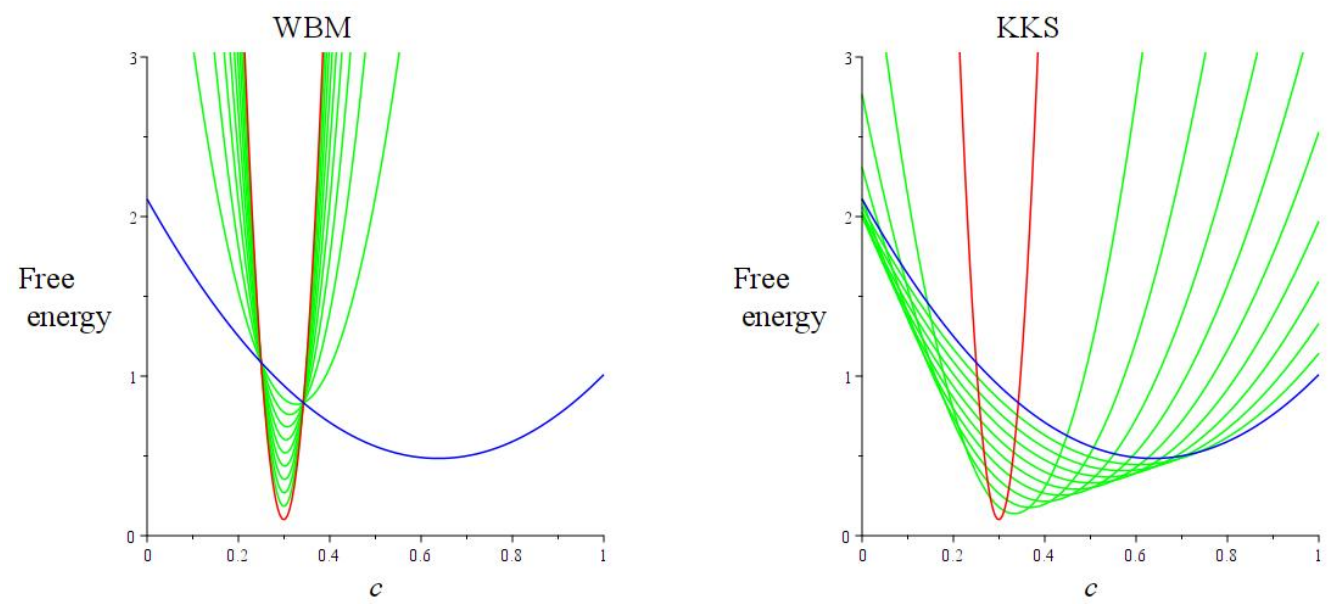

Figure 9. (Left): WBM method of forming the bulk free energy, $f_{B}$ from the pure phases shown in red and blue. (Right): KKS method of forming the bulk free energy, $f_{B}$ from the pure phases shown in red and blue. The red curve (seen more clearly in this plot) represents an intermetallic with a narrow range of possible mixes. In both plots the intervening phases, shown in green are for mixture of the pure phases found in the transition region - values of $\phi=0.1,0.2, \ldots 0.9$

Either way, both methods still present an unresolved problem, for which this section puts forward a solution, applicable for both (and any) interpolation methods. A key parameter in phase-field modelling is $\delta$, the interface width. The value of $\delta$ may be given a physically real value, possibly arising from a molecular dynamic simulation. However, often the value is dictated by making it small enough to capture features of the surface morphology. A physically real interface width usually implies that there is a very different characteristic length associated with the solute diffusion as compared with the interface width, resulting in many more grid points, less computational stability and significantly longer run times. Thus, a larger interface width, say, of the same order as the concentration length associated with diffusion (note, this also depends on growth speed) is computationally convenient. It then becomes essential that the influence of interface width on results must be addressed. Note that this model-induced 'solute trapping' effect is different to physical solute trapping, as studied in [26].

Writing the two-phase free energy, Equation (29) in 1D we have

$$
f=\frac{1}{2} A^{2} \delta^{2}\left(\frac{\partial \phi}{\partial x}\right)^{2}+\Omega(\phi)+g(\phi) f_{\text {liq }}(c)+(1-g(\phi)) f_{\text {liq }}(c)
$$

where $\Omega$ is the double-well potential, e.g., $\Omega=\frac{1}{2} \phi^{2}(1-\phi)^{2}$, and derive equations of motion

$$
\dot{\phi}=M\left[\delta^{2} \frac{\partial^{2} \phi}{\partial x^{2}}-g^{\prime}(\phi)\left(f_{\text {liq }}-f_{\text {sol }}\right)\right]
$$

and

$$
\dot{c}=\nabla \cdot D \nabla f_{c}=\partial_{x}\left(D \partial_{x} f_{c}\right)
$$

where $f_{c} \equiv \frac{\partial f}{\partial c}$. We now assume that we have a phase profile of the form

$$
\phi=\frac{1}{1+\exp [-(x-V t) / \delta]}
$$


that solves the 1D equation, Equation (31) for some $V$ and $\delta$. Note that the slope of the phase-field is related to the interface width by

$$
\left.\frac{\partial \phi}{\partial x}\right|_{x=0}=\frac{1}{4 \delta} .
$$
that

For the profile, Equation (33), moving at constant speed, $V$, we have, $\frac{\partial}{\partial t}=-V \frac{\partial}{\partial x}$ so

$$
-V \partial_{x} c=\partial_{x}\left(D \partial_{x} f_{c}\right)
$$

or

$$
\partial_{x}\left(V c+D \partial_{x} f_{c}\right)=0
$$

Now, $f_{c}$ is of generic form and phase-dependent; therefore, given the boundary conditions for Equation (36), it does not have a ready solution. On applying the numerical solution, we find that we can fix the value of the solute in the solid and at infinity, but that the value in the interface arises from the solution-see Figure 10, which shows a value for $c_{L}$ arising in the solution of the 1D problem for $\delta=2$ of $c \approx 0.6$ and for $\delta=0.2$ of $c \approx 0.4$-both of which may be compared with the equilibrium value (or equivalently $\delta \rightarrow 0)$ of $c=c_{L}^{E}(=0.35)$.

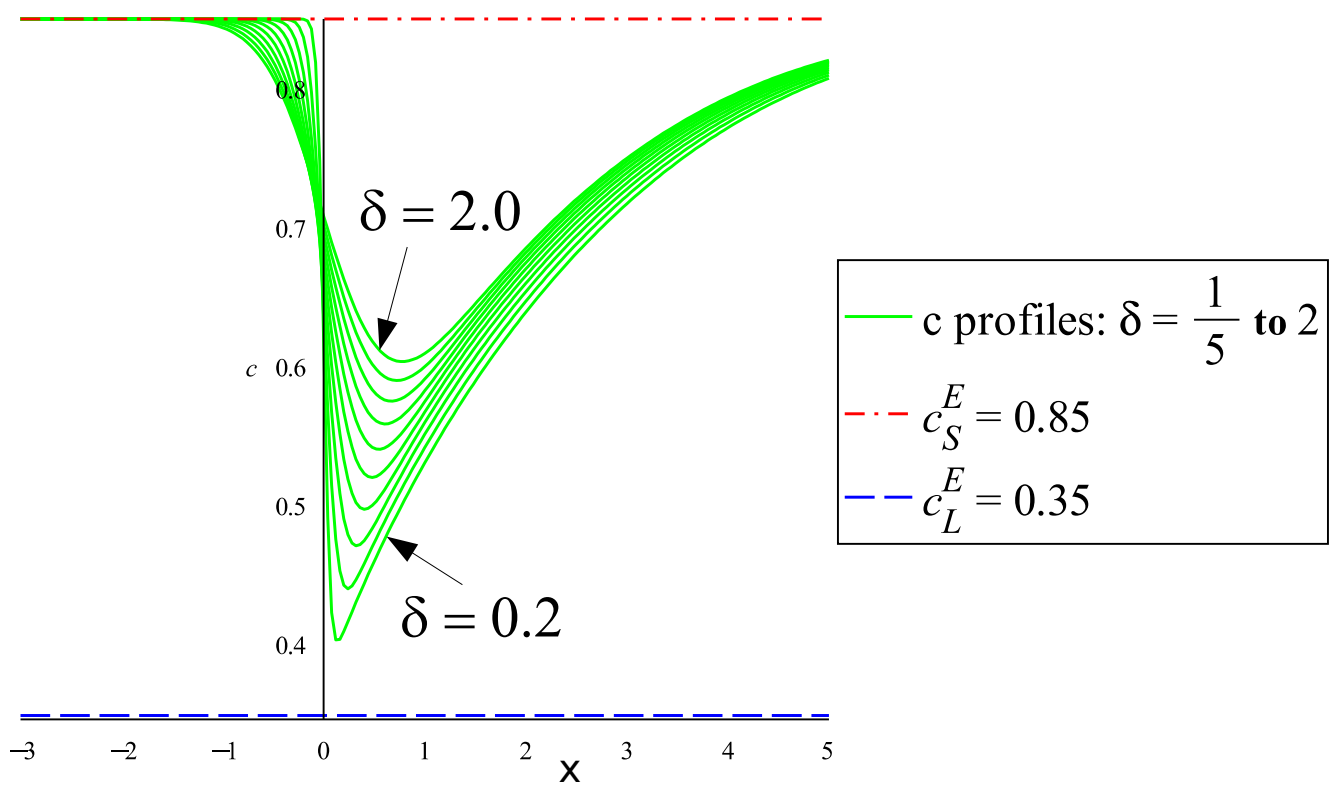

Figure 10. Example 1D steady state solutions to Equation (36), for $V / D=5$ for a given free energy, with various phase-field interface widths, $\delta=\frac{1}{5}, \frac{2}{5}, \ldots, 2$. None of the solutions here give the liquid equilibrium minimum, $c_{L}^{E}=0.35,(\delta=1 / 5$ is the closest, at $\sim 0.4)$.

For two convex free energy curves, $f_{\text {liq }}$ and $f_{\text {sol }}$, there is a unique point on each curve such that

$$
\frac{f_{\mathrm{liq}}\left(c_{L}\right)-f_{\mathrm{sol}}\left(c_{S}\right)}{c_{L}-c_{S}}=f_{\mathrm{liq}}^{\prime}\left(c_{L}\right)=f_{\mathrm{sol}}^{\prime}\left(c_{S}\right)
$$

Therefore, by choosing $c_{S}$ as one boundary value, we find, as $V$ decreases, that the extreme value in the interface, $c_{L}$ tends towards the common tangent value for the other function, $f_{\text {liq. }}$. However, as shown in Figure 10, for $V>0$ the result becomes dependent on $\delta \in[0.2,2.0]$ with the minimum value of the solute profile falling short of the required 
value of $c_{L}^{E}$ (assuming $c_{L}^{E}<c_{S}^{E}$ ). In [27], we show that it is possible to modify Equation (36) to the form

$$
\partial_{x}\left(V c+D \partial_{x} f_{c}+D \alpha\right)=0
$$

and demand that $\alpha$ is chosen such that $c_{L}=c_{L}^{E}$, for a given $\delta$ and $V$. By inspecting the dependency of $\alpha$ on both $V$ and $\delta$, we find that the dependence is well approximated by

$$
\alpha=\beta V \delta f_{c \phi} \phi^{\prime}(x) / D_{L}
$$

for some constant, $\beta$. In other words, finding $\beta$ gives an anti-trapping current across a range of velocities, $V$, and interface widths, $\delta$. We illustrate this in Figure 11 for a range $\delta=0.1$ to 1 , where $\beta$ is chosen so that $\alpha$ is exact for $\delta=1$.

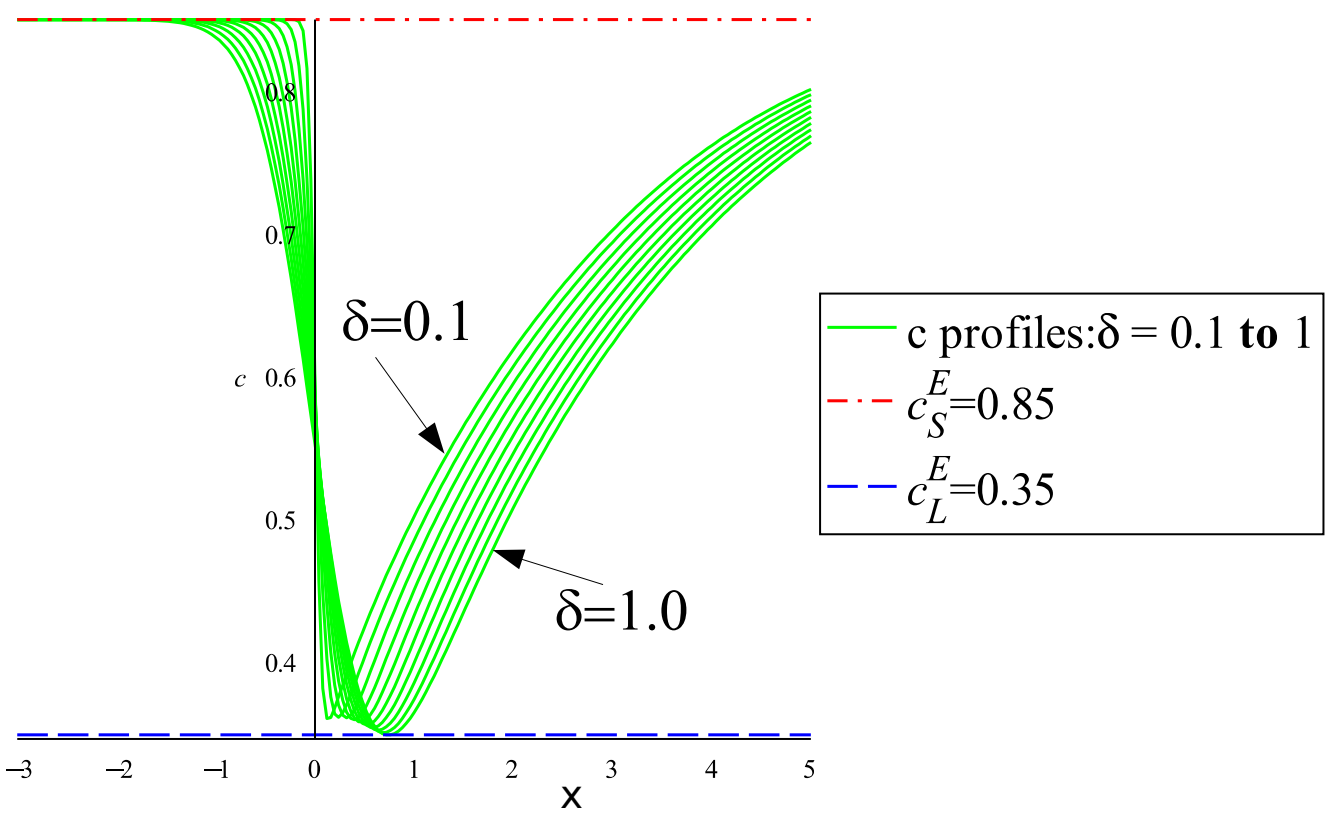

Figure 11. Solutions to the same steady state 1D problem as illustrated in Figure 10 but with an anti-trapping current. The value for $\delta=1$ is chosen to be exact by the choice, and for $\delta<1$, the anti-trapping is approximate.

Using the value of $V$ and $\delta$ at the tip of a growing dendrite in 2D or 3D, together with $\beta$, then allows us to define a compensating current for use in two and three dimensions:

$$
\mathbf{j} \equiv-\frac{D}{D_{L}} f_{c \phi} \beta \delta V \nabla \phi
$$

to be used with a modified solute equation:

$$
\dot{c}=\nabla \cdot\left(D \nabla f_{c}+\mathbf{j}\right)
$$

Applying this model with physically realistic functions for liquid and solid phase PbSn, we can extract the partition coefficient $c_{\text {sol }} / c_{\text {liq }}$ from the $c$ field at the tip of a growing dendrite and compare with the equilibrium value and with changes in interface width $\delta$. A plot of results is shown in Figure 12, where the dotted lines are without anti-trapping and the solid lines with the current in Equation (41). The latter shows remarkable interface width independence and agreement with the equilibrium value. Without ant-trapping, the solid lines are too high, indicating that the value of $c_{\text {liq }}$ is too high. Please note that concentration in a binary system, $A-B$, may define $c$ as the amount of $A$ or the amount of $B$. Thus, depending on convention, we may have $c_{L}^{E}<c_{S}^{E}$ or $c_{L}^{E}>c_{S}^{E}$. Thus, in Figures 10 and 11, 
we have implicitly adopted $c_{L}^{E}<c_{S}^{E}$, but in the result, Figure 12, we have used the opposite convention.

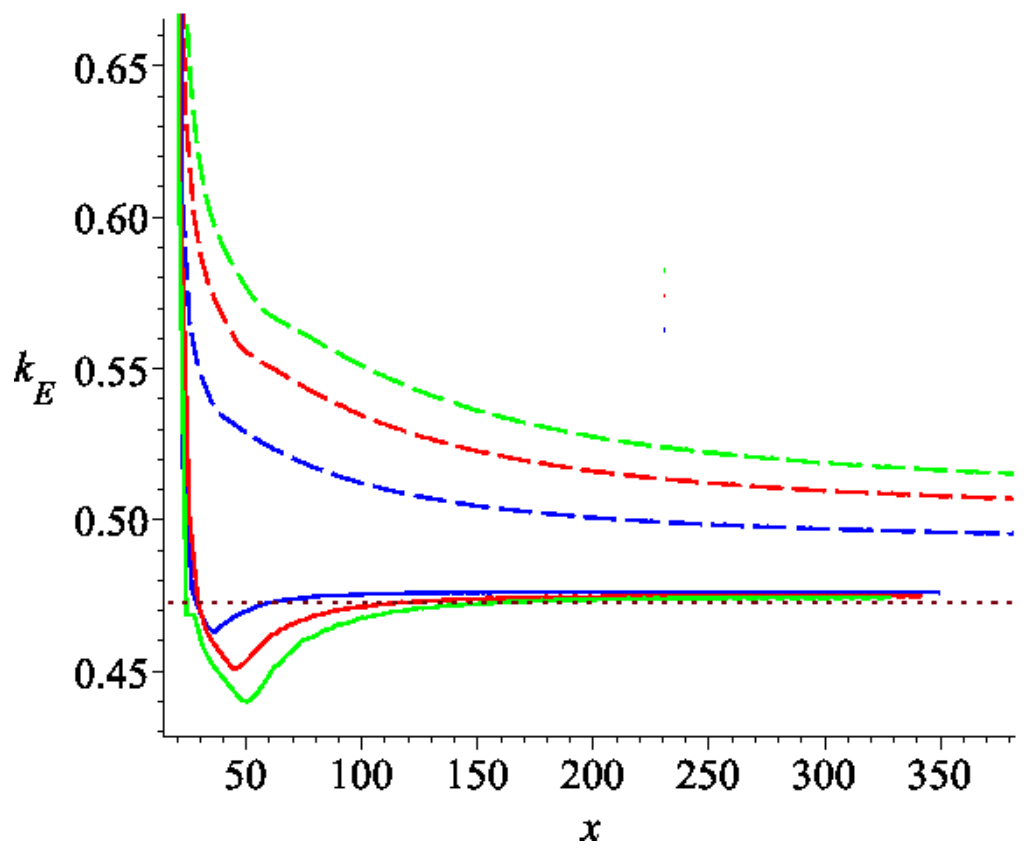

Figure 12. Here, the lines represent the value $k_{E} \equiv c_{\mathrm{sol}} / c_{\text {liq }}$ at a growing tip for three values of $\delta$, for both the standard simulation (dashed), and the simulation with an anti-trapping current (solid), the interface-width, $\delta$, is for a range with the largest (green) being twice that of the smallest (blue), and red being intermediate: $\delta / \sqrt{8}=4,6,8$,

\section{Eutectic Modeling}

A eutectic involves two solid phases growing symbiotically such that the growth of one solid phase reduces solute favourable to its growth, whilst leaving behind conditions favourable for growth of the other solid phase. Unlike single-phase growth, there is less of a build up of concentration at the growing surface. Initial models, e.g., [28], for multiphase were, in analogy with the single-phase model, of the form

$$
\dot{\phi}_{i}=-M \frac{\delta F}{\delta \phi_{i}}, i=1 \ldots n
$$

for $n$ phases and given some suitable form for free energy density. However, this form of the equations must be used in conjunction with a Lagrange multiplier (see also [28]) to avoid the constraint

$$
\sum_{i}^{n} \phi_{i}=1
$$

being violated. When a Lagrange multiplier is used, it turns out that the form of the equations becomes

$$
\dot{\phi}_{i}=-M_{i j} \frac{\delta F}{\delta \phi_{j}}
$$

where the square matrix, $\mathbf{M}$ is given by

$$
\mathbf{M}=M\left[\begin{array}{rrr}
2 & -1 & -1 \\
-1 & 2 & -1 \\
-1 & -1 & 2
\end{array}\right]
$$


for some constant $M$. This guarantees that

$$
\sum_{i}^{n} \dot{\phi}_{i}=0
$$

and so with consistent initial conditions, Equation (43) is satisfied for all time, $t$. Unfortunately, such a mobility matrix, Equation (45), and its generalisation to $n>3$ fails to reduce properly when only two of the $n>2$ phases are present. A spurious phase will likely be generated. This is because, for example, with $\phi_{1}=0$ :

$$
\dot{\phi}_{1}=-2 \frac{\delta F}{\delta \phi_{1}}+\frac{\delta F}{\delta \phi_{2}}+\frac{\delta F}{\delta \phi_{3}}
$$

the contribution from the second and third terms can be nonzero. An alternative formulation, that we propose in [29], is to specify the mobility matrix as phase-dependent

$$
M_{i j}=-\frac{\phi_{i} \phi_{j}}{\left(1-\phi_{i}\right)\left(1-\phi_{j}\right)}, i \neq j
$$

and

$$
M_{i i}=-\sum_{j \neq i} M_{i j} .
$$

To understand this form of matrix, it helps to note that when only two phases are present, the multiphase equations become identical to the single-phase equations. Furthermore, if there are equal amounts of each phase, the matrix reduces to a multiple of Equation (45) (used in Equation (44)). An illustration of the mobility matrix is given in Figure 13 for three phases. At the triple point, $\phi_{1}=\phi_{2}=\phi_{3}, \mathbf{M}$, reduces to a multiple of the matrix given at (45), and we find that

$$
\mathbf{M}\left[\begin{array}{l}
v_{1} \\
v_{2} \\
v_{3}
\end{array}\right]=\lambda\left[\begin{array}{l}
v_{1} \\
v_{2} \\
v_{3}=-v_{1}-v_{2}
\end{array}\right]
$$

for some scalar, $\lambda$. If $\mathbf{v}=\left[v_{1}, v_{2},-v_{1}-v_{2}\right]$ then $\mathbf{M v}=\lambda \mathbf{v}$, and we represent the matrix, $\mathbf{M}$, as a circle since it operates on any vector to return a multiple of the vector in the same direction. Similarly, on the edge (but not at the vertices), Mv returns a vector pointing along the edge, e.g., for $\phi_{3}=0$ we have

$$
\mathbf{M}\left[\begin{array}{l}
v_{1} \\
v_{2} \\
v_{3}
\end{array}\right]=\lambda\left[\begin{array}{l}
v_{1} \\
v_{2}=-v_{1} \\
v_{3}=0
\end{array}\right]
$$

which we represent as a degenerate ellipse appearing as a line tangential to the edge in Figure 13. The intermediate cases are shown as intermediate ellipses. An important case is when $\left[\phi_{1}, \phi_{2}, \phi_{3}\right]$ is near a vertex, e.g., $\left[\phi_{1}=1-2 q, \phi_{2}=q, \phi_{3}=q\right]$ for $q \ll 1$. In this latter case, the matrix is represented by the ellipse on the bottom left of the simplex in Figure 13. 


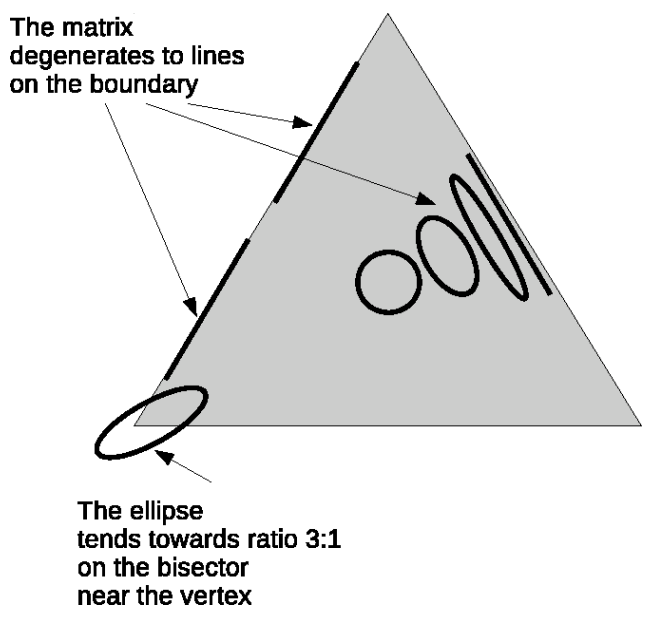

Figure 13. An illustration of the action of the mobility matrix given by Equation (48). For comparison, the Lagrange multiplier approach would have every part of the region depicted as a circle (as in the centre of the triangle). On each edge, the two-phase regions, the ellipses become degenerate.

For single phase, the formation of the surface energy density for isotropic growth is given by Equation (18). Therefore, it is natural to postulate a multiphase surface energy density by the equation postulated in [28]:

$$
f_{S}=\sum_{i=1}^{n} \frac{1}{2} \delta^{2} \nabla \phi_{i} \cdot \nabla \phi_{i}+\frac{1}{2} \phi_{i}^{2}\left(1-\phi_{i}\right)^{2}
$$

where, indeed, this form reduces correctly when only two phases are present. As recognised at the time by the authors of [28], the form in Equation (52) is not sufficiently general because the angles at the eutectic triple junction are enforced to be equal to $120^{\circ}$. This is insufficiently general since the inter-facial tensions between the three pairs of phases are usually unequal, and there is no provision (in Equation (52)) for the binary interaction of the surface energy between two phases. Another approach, which at least allows interfacial energies, $\sigma_{i j} \propto \delta_{i j}$, to be included, and also consistent with two phases, was proposed in $[30,31]$ involving a double sum:

$$
f_{S}=\sum_{i=1}^{n} \sum_{j \neq i}^{i-1} \frac{1}{2} \delta_{i j}^{2}\left|\phi_{i} \nabla \phi_{j}-\phi_{j} \nabla \phi_{i}\right|^{2}+\frac{1}{2} \phi_{i}^{2} \phi_{j}^{2} .
$$

It is not clear that the formulation Equation (53) does actually control the angle at the junction. Some formulations introduce angle control by introducing an extra potential in the form of (for three phases), $\phi_{1} \phi_{2} \phi_{3}$ or its square [7]. In [32], we propose a new formulation, which gives direct control of the angle at the triple junction:

$$
f_{S}=\sum_{i=1}^{n} \frac{1}{2} \delta_{i}^{2} \nabla \phi_{i} \cdot \nabla \phi_{i}+\frac{1}{2} \phi_{i}^{2}\left(1-\phi_{i}\right)^{2}
$$

where as a consequence of a definition

$$
\delta_{i j}^{2}=\delta_{i}^{2}+\delta_{j}^{2}
$$

the single indexed quantities may be written as a function of the double indexed quantities by

$$
\delta_{i}^{2}=\delta_{i j}^{2}+\delta_{i k}^{2}-\delta_{j k}^{2}, i \neq j \neq k
$$

Here, $\delta_{i j}, i \neq j$ is the surface energy between phase $\phi_{i}$ and $\phi_{j}$. The relation of the surface energy to the angle at the triple junction is shown in Figure 14, which shows three lines 
containing three desired angles and then a triangle such that each side, $n_{i j}$ crosses the lines at right angles. The relative lengths of the triangle sides are proportional to the quantities, $\delta_{i j}$. To obtain a feel for the formulation, set the two solid interface widths $\delta_{2}=\delta_{3}=1$, then when $\delta_{1}$ (the liquid) is equal to unity the formulation, Equation (52) is returned, but when $\delta_{1}>1$, the angle in the liquid area begins to increase past $120^{\circ}$, with the other two equal regions decreasing accordingly.

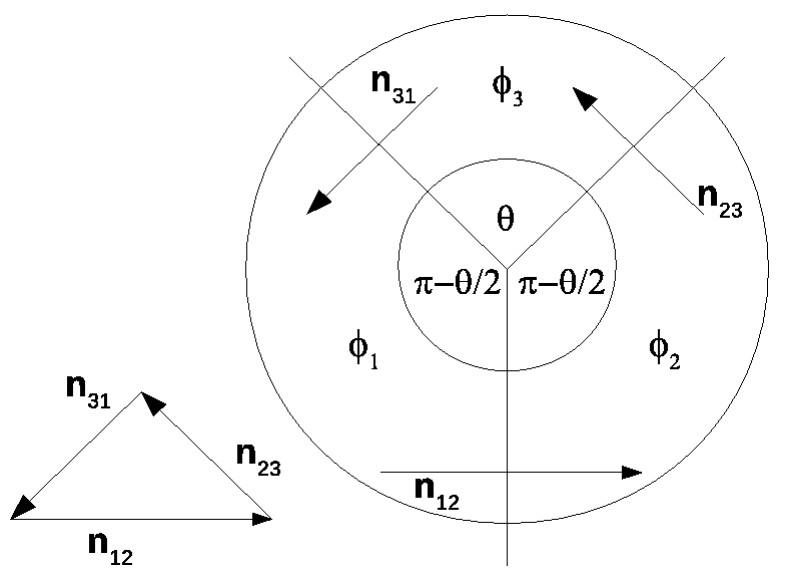

Figure 14. The relationship between junction angle and surface energy. These binary indexed proportions are then used to construct a single index, which enters the surface energy term in the phase-field.

Using a surface energy defined by Equation (54) in phase-field gives the desired angle control, as shown in Figure 15. 


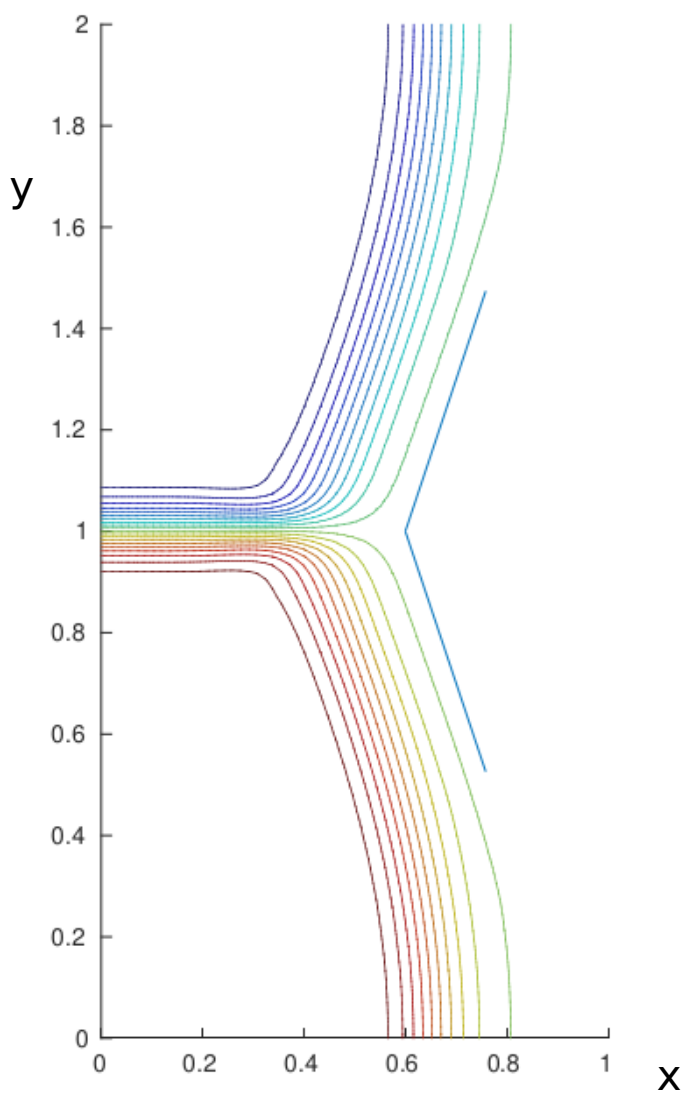

Figure 15. The contours of the phase-field align with the desired angle (here $143^{\circ}$, shown as two lines, coloured blue, slightly to the right of the contours ). Here, the growth of the eutectic is in the $x$-direction, with the partition between the two solids being at $y=1$.

The construction of the multiphase model was greatly aided by the postulation, in Section 5 of [32], of a triple junction field. Here, we use the normals to the three boundaries (see Figure 14), $\mathbf{n}_{12}, \mathbf{n}_{23}$ and $\mathbf{n}_{31}$ to form the three phase fields

$$
\begin{aligned}
& \phi_{1}=\frac{1}{1+\exp \left(\mathbf{x} \cdot \mathbf{n}_{12}\right)+\exp \left(-\mathbf{x} \cdot \mathbf{n}_{31}\right)} \\
& \phi_{2}=\frac{1}{1+\exp \left(\mathbf{x} \cdot \mathbf{n}_{23}\right)+\exp \left(-\mathbf{x} \cdot \mathbf{n}_{12}\right)} \\
& \phi_{3}=\frac{1}{1+\exp \left(\mathbf{x} \cdot \mathbf{n}_{31}\right)+\exp \left(-\mathbf{x} \cdot \mathbf{n}_{23}\right)}
\end{aligned}
$$

From $\mathbf{n}_{12}+\mathbf{n}_{23}+\mathbf{n}_{31}=\mathbf{0}$, the constraint, $\sum_{i=1}^{3} \phi_{i}=1$ is automatically satisfied. Using these fields allows inspection of the two terms in the surface energy - the gradient as well as the potential well. This also allows comparison with other postulated models, as shown in Figure 16, where the surface energy at the centre of the simplex, $\phi_{i}=1 / 3, i=1,2,3$, is lower than at the binary junctions on the left but, conversely, higher for the model advocated in our paper [32]. 

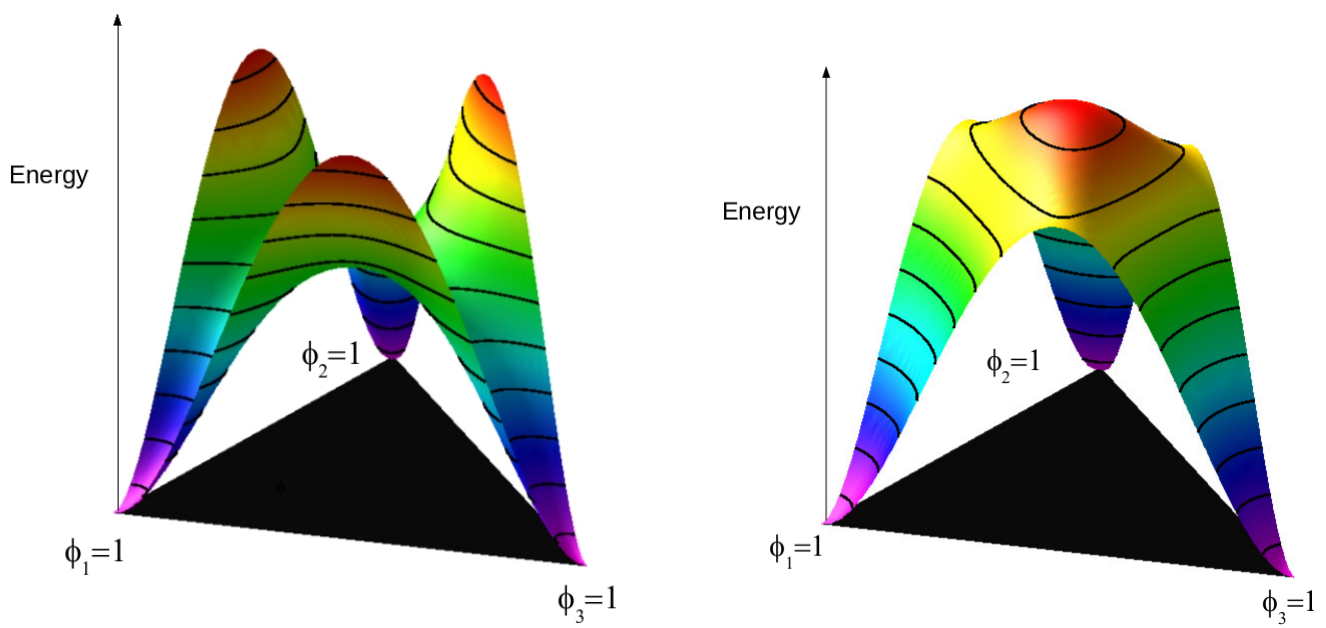

Figure 16. The two plots show the energy over a simplex as a function of three phrases, $\phi_{1}, \phi_{2}, \phi_{3}$ (colour is coincident with the height). The centre of the simplex is formally the triple junction point where, ideally, the surface energy barrier should be highest. The left plot shows the models advocated by [30,31] containing a hollow at the junction, whereas the model proposed by [32] (and also [28] in the special case of equal angles) has the formally correct barrier at the triple point as well as the binary junctions.

The other feature present in our model (the right of Figure 16) is that the gradient of surface energy is zero at the boundary of the simplex, but this is not the case in the models advocated in $[30,31]$. A low triple junction barrier generates a spurious phase at a binary junction. The model advocated in [32] avoids both spurious phase generation and also, due to the zero gradient, does not give preference to a value outside the simplex, i.e., non-physical negative values of $\phi_{i}$. It is interesting to note that the use of a potential well known as the double obstacle, given by

$$
\Omega=\sum_{i \neq j} \phi_{i} \phi_{j}
$$

helps to mitigate against spurious phase growth by presenting a high potential at the triple point. Its single-phase counterpart given by

$$
\Omega=\phi(1-\phi)
$$

has an intrinsic driving force on $\phi$ to be outside its range [0,1], a feature that is inherited for multiphase in Equation (58). Hence, the handling in computer code needs special care, where one reward for doing so is that the interface region in a double obstacle code is finite, allowing reduced degrees of freedom for the phase variable that is formally a unit or zero finite distance away from the transition region. Our approach to this is guided by the underlying philosophy behind the phase field, that is, smoothness and continuity, and hence, a preference for Equation (54). See also [33] for a comparison of potentials for phase field.

\section{Fully Coupled Solute and Temperature Multiphase Modelling}

As stated in the introduction, the early days of phase-field solidification focussed on pure metal solidification, and the movement towards the more interesting, from a practical engineering perspective, complex alloy solidification came later. Because of the high conductivity of metals, the temperature field can legitimately be neglected in many scenarios. That said, there are situations where the temperature field plays a crucial role in alloy solidification, such as growth restriction [34,35], but also when the partition coefficient, $k_{E} \rightarrow 1$ (e.g., for almost complete solute trapping at a very high growth rate), whence thermal forces exceed diffusion effects. One way of processing this is to impose a 
temperature field on the other fields, but this does not take into account the heat generation due to the volume latent heat of fusion.

In the early 2000s, fully coupled single-phase formulations were in existence [3,9], but the more general fully coupled multiphase formulation did not. A general multiphase framework for the formulation of thermodynamically consistent phase-field models was in demand, principally to provide a temperature field equation in this more general setting. The difficulty of extending a temperature formulation from single to multiphase-field can be seen by inspecting the single-phase contribution to the temperature diffusion equation,

$$
C_{v} \dot{T}=\nabla \cdot k \nabla T+L \dot{\phi},
$$

where $C_{v}$ is heat capacity at constant volume, $\kappa$ conductivity and $L$ the latent heat. The term $L \dot{\phi}$ generates heat as the material changes phase. Now consider a plausible multiphase generalisation given by

$$
C_{v} \dot{T}=\nabla \cdot k \nabla T+L \sum_{i}^{n} \dot{\phi}_{i} .
$$

This fails because $\sum_{i}^{n} \dot{\phi}_{i}=0$. A compromise might be to single out the liquid phase as special and write

$$
C_{v} \dot{T}=\nabla \cdot k \nabla T+L \dot{\phi}_{\text {liquid }}
$$

but this undermines an implicit assumption in phase-field modelling that no phase is special-even the liquid. The other issue arising in this investigation was to question the single-phase temperature equation itself. Do heat capacity and latent heat need adding to the model or do they arise naturally from the free energy? Why, in the case of binary alloys, do we not require heat generation due to solute change?

A similar set of problems arose in the modelling of complex fluids where a typical approach to model these fluids at the time was to specify the viscosity and stress tensor in terms of velocity gradients in a manner inspired by spring and dash-pot analogies, see [36]. These models and the concern that the resulting models may not be thermodynamically consistent was addressed in [8], which proposed a formalism for modelling complex fluids that closely resembled phase-field models of solidification. That is, to start the modelling by a specification of the energy and deduce the evolution equations using a modification of the Poisson bracket for continuum mechanics. We see in [37] that it turns out that one of the simplest examples of the operation of bracket formalism is the purely dissipative phase-field models of solidification-particularly, the isothermal single and multiphase phase models. The formalism also shows how to recover the entropy from which it is a short step to generating a temperature equation. Then it becomes clear that the only additional parameter required to specify a temperature equation is the conductivity, $k$. Not only is the heat capacity and latent heat prescribed (from the free energy specification), but the addition of a term involving $\dot{c}$, similar to the latent heat of fusion, also falls out of the formalism, and treatment of density change together with necessary fluid flow is also prescribed.

The n-phase formulation for the temperature field of a binary alloy, postulated in [37] is given as:

$$
C_{v} \dot{T}=\nabla \cdot \mathbf{q}+\sum_{i=1}^{n} L_{i} \dot{\phi}^{i}+K \dot{c}
$$

where

$$
C_{v} \equiv-T \frac{\partial}{\partial T} \frac{\partial f}{\partial T}
$$




$$
\begin{gathered}
L_{i} \equiv-\left(1-T \frac{\partial}{\partial T}\right) \frac{\delta F}{\delta \phi_{i}} \approx-\left(1-T \frac{\partial}{\partial T}\right) \frac{\partial f_{B}}{\partial \phi_{i}} \\
K \equiv-\left(1-T \frac{\partial}{\partial T}\right) \frac{\partial f_{B}}{\partial c} \\
\mathbf{q} \equiv \kappa \nabla T+D \frac{\partial f_{B}}{\partial c} \nabla \frac{\partial f_{B}}{\partial c}
\end{gathered}
$$

where $D$ is the solute diffusivity. If we neglect the contributions due to solute change and gradients, we have

$$
C_{v} \dot{T}=\nabla \cdot \kappa \nabla T+\sum_{i=1}^{n} L_{i} \dot{\phi}^{i}
$$

This, with hindsight, seems a reasonable guess, but Equation (65) is required to complete the definition, not only making the latent heat terms, $L_{i}$, non-constant but even differing in sign.

Finally, the general bracket formalism also poses the question as to why there is no further coupling between phase and solute concentration as would naturally occur if solute, $c$ and the phase-fields were treated equally as part of a general vector $\boldsymbol{\phi}=\left[c, \phi_{1}, \phi_{2}, \phi_{3}\right]$, something that has been noted and used by other authors, such as [38]. The key novel part of which is that one constructs the bracket alongside the free energy. The bracket includes the diffusion parameters, which fields are conserved or not, and draws a distinction between energy conserving fields such as mass, and the dissipative dynamics as is the staple of relaxation phenomena such as solidification.

\section{Summary}

Here, we summarise our main contributions to phase-field computational modelling.

1. Nonlinear adaptive mesh multigrid solver for multi-scale problems [10].

2. Load balancing techniques for parallel solvers [11].

3. Control of phase-field profile to match molecular dynamic or ab initio calculations [14].

4. Transition from faceted (equilibrium) to dendritic morphology (non-equilibrium) [21].

5. A general approach to formulate arbitrary faceted polyhedrons and polyhedra in phase-field [17].

6. A method to give interface width independence for models using arbitrary free energy functions in single-phase simulations [27].

7. Control of triple junction angle in eutectic growth [32].

8. Development of a broader formalism to give a thermodynamically consistent phasefield formulation with correct thermal field and entropy generation [37].

\section{Appendix A. Non-Dimensional Phase-Field Equations}

Both the phase-field parameter, $\phi$, and concentration, $c$, are dimensionless but free energy density functions provided from data bases, e.g., [39], are typically in units of Joules per mole. From Equations (3) and (5), a fully dimensioned form of the two-phase-field equation is of the form

$$
\dot{\phi}=-M \frac{\delta F}{\delta \phi}=M\left[\nabla \cdot \frac{\partial f_{S}}{\partial \nabla \phi}-\frac{\partial f_{B}}{\partial \phi}\right]
$$


implying that the units of $M$ are Moles per (Seconds $\times$ Joules $\times$ volume), or $\mathrm{Mol} /\left(\mathrm{s} \mathrm{Jm}^{3}\right)$ is S.I. units. Taking the isotropic model (see Equation (9) for comparison) as a template for the non-dimensional procedure:

$$
\begin{aligned}
\dot{\phi} & =M\left[W\left(\delta^{2} \nabla^{2} \phi-\Omega^{\prime}(\phi)\right)-f_{B}^{\prime}(\phi)\right] \\
& =M W \delta^{2}\left[\nabla^{2} \phi-\frac{\Omega^{\prime}(\phi)}{\delta^{2}}-\frac{f_{B}^{\prime}(\phi)}{W \delta^{2}}\right]
\end{aligned}
$$

where $\Omega \equiv \frac{1}{2} \phi^{2}(1-\phi)^{2}$ is a typical choice for the double-well potential, and $W$ is associated with surface energy with units adjusted to be the same as $f_{B}$. In the simplest models, the parameters, $M, W$ and $\delta$ are all constants. Moreover, $M W \delta^{2}$ has the units of diffusivity and can conveniently be used as the unit of diffusivity, $M W \delta^{2} \equiv D_{\text {char }}$. Writing the ratio $f_{B} / W$ as $\bar{f}_{B}$ and using $\delta$ as the unit of length reduces the fully dimensional equation to the non-dimensional form:

$$
\dot{\phi}=\nabla^{2} \phi-\Omega^{\prime}(\phi)-\bar{f}_{B}^{\prime}(\phi)
$$

This then gives the non-dimensional concentration equation as

$$
\dot{c}=\nabla \cdot \bar{D} \nabla \bar{f}_{B}^{\prime}(c)
$$

where

$$
\bar{D}=\frac{D}{D_{\text {char }}} .
$$

Using these two coupled equations, we can explore a range of parameter values: $M$, the mobility (response of the front to the driving force); $W$, the coupling between surface and bulk forces; $\delta$, the interface width, which may vary between physically-based and computationally convenient. Alternatively, one may fix all the constants by reference, if possible, to physically determined parameters, and then vary $f_{B}$ as a function of temperature, $T$, to explore aspects of morphology as a function of undercooling.

Author Contributions: Conceptualisation, P.C.B., P.K.J., A.M.M.; investigation, writing-original draft preparation, methodology, P.C.B.; writing-review and editing, supervision, P.K.J. and A.M.M.; funding acquisition, project administration A.M.M. All authors have read and agreed to the published version of the manuscript.

Funding: This research was funded by the EPSRC Innovative Manufacturing Research Hub in Liquid Metal Engineering (LiME), Grant No. EP/ N007638/1.

Acknowledgments: We thank the journal publishers for permission to reprint the figures from our original articles that we explicitly cite.

Conflicts of Interest: The authors declare no conflict of interest. The funders had no role in the design of the study; in the collection, analyses, or interpretation of data; in the writing of the manuscript, or in the decision to publish the results.

\section{References}

1. Karma, A.; Rappel, W. Quantitative phase-field modeling of dendritic growth in two and three dimensions. Phys. Rev. E 1998, 57, 4323-4349.

2. Karma, A. Phase-Field Formulation for Quantitative Modeling of Alloy Solidification. Phys. Rev Lett. 2001, 87-11, 115701.

3. Ramirez, J.C.; Beckermann, C.; Karma, A.; Diepers, H.J. Phase-field modeling of binary alloy solidification with coupled heat and solute diffusion. Phys. Rev. E 2004, 69, 051607. https://doi.org/10.1103/PhysRevE.69.051607.

4. Boettinger, W.J.; Warren, J.A.; Beckermann, C.; Karma, A. Phase-Field Simulation of Solidification. Annu. Rev. Mater. Res. 2002, 32, 163-194, https://doi.org/10.1146/annurev.matsci.32.101901.155803.

5. Steinbach, I. Why Solidification? Why Phase-Field? JOM 2013, 65, 1096-1102. 
6. Moelans, N.; Blanpain, B.; Wollants, P. An introduction to phase-field modeling of microstructure evolution. Calphad Comput. Coupling Phase Diagrams Thermochem. 2008, 32, 268-294.

7. Toth, G.I.; Pusztai, T.; Granasy, L. Consistent multiphase-field theory for interface driven multidomain dynamics. Phys. Rev. $B$ 2015, 92, 184105.

8. Beris, A.N.; Edwards, B.J. Thermodynamics of Flowing Systems; Oxford University Press: New York, NY, USA, 1994.

9. Rosam, J.; Jimack, P.; Mullis, A. An adaptive, fully implicit multigrid phase-field model for the quantitative simulation of non-isothermal binary alloy solidification. Acta Mater. 2008, 56, 4559-4569. https://doi.org/10.1016/j.actamat.2008.05.029.

10. Bollada, P.C.; Goodyer, C.E.; Jimack, P.K.; Mullis, A.M.; Yang, F.W. Three Dimensional Thermal-Solute Phase Field Simulation of Binary Alloy Solidification. J. Comp. Phys. 2015, 287, 130-150.

11. Chen, M.H.; Bollada, P.; Jimack, P. Dynamic Load Balancing for the Parallel, Adaptive, Multigrid Solution of Implicit Phase-Field Simulations. Int. J. Numer. Anal. Model. 2019, 16, 297-318.

12. Yang, F.W.; Goodyer, C.E.; Hubbard, M.E.; Jimack, P.K. An optimally efficient technique for the solution of systems of nonlinear parabolic partial differential equations. Adv. Eng. Softw. 2017, 103, 65-84.

13. Atkins, M.R. A Method of Calculating the Thickness of a Solid-Liquid Interface. 2010. https://doi.org/10.1137/10s010636.

14. Bollada, P.; Men, H.; Fang, C.; Jimack, P.; Fan, Z.; Mullis, A. A novel route to the coupling of molecular dynamics and phase-field simulations of crystal growth. IOP Conf. Ser. Mater. Sci. Eng. 2019, 529, 012032. https://doi.org/10.1088/1757899X/529/1/012032.

15. Ohno, M.; Takaki, T.; Shibuta, Y. Numerical testing of quantitative phase-field models with different polynomials for isothermal solidification in binary alloys. J. Comput. Phys. 2017, 335, 621-636.

16. Takaki, T.; Sakane, S.; Ohno, M.; Shibuta, Y.; Shimokawabe, T.; Aoki, T. Primary arm array during directional solidification of a singlecrystal binary alloy: Large-scale phase-field study. Acta Mater. 2016, 118, 230-243. https://doi.org/10.1016/j.actamat.2016.07.049.

17. Bollada, P.C.; Jimack, P.K.; Mullis, A.M. A vertex based approach to crystal facet modelling in phase field. Comput. Mater. Sci. 2021, 192, 110331

18. Salvalaglio, M.; Backofen, R.; Bergamaschini, R.; Montalenti, F.; Voigt, A. Faceting of Equilibrium and Metastable Nanostructures: A Phase-Field Model of Surface Diffusion Tackling Realistic Shapes. Cryst. Growth Des. 2015, 15, 2787-2794, doi:10.1021/acs.cgd.5b00165.

19. Eggleston, J.; McFadden, G.; Voorhees, P. A phase-field model for highly anisotropic interfacial energy. Phys. D Nonlinear Phenom. 2001, 150, 91-103. https://doi.org/10.1016/S0167-2789(00)00222-0.

20. Yuan, X.F.; Liu, B.Y.; Li, C.; Zhou, C.S.; Ding, Y.T. Simulation of facet dendrite growth with strong interfacial energy anisotropy by phase field method. J. Cent. South Univ. 2015, 22, 855-861. doi:10.1007/s11771-015-2593-8.

21. Bollada, P.; Jimack, P.; Mullis, A. Faceted and dendritic morphology change in alloy solidification. Comput. Mater. Sci. 2018, 144, 76-84. https://doi.org/10.1016/j.commatsci.2017.12.007.

22. Wheeler, A.; Boettinger, W.; McFadden, G. Phase-field model for isothermal phase transitions in binary alloys. Phys. Rev. A 1992, 45, 7424-7440.

23. Choudhury, A.; Kellner, M.; Nestler, B. A method for coupling the phase-field model on a grand-potential formalism to thermodynamic databases. Curr. Opin. Solid State Mater. Sci. 2015, 19, 287-300.

24. Kim, S.G.; Kim, W.T.; Suzuki, T. Phase-field model for binary alloys. Phys. Rev. E 1999, 60, 7186-7197.

25. Plapp, M. Unified derivation of phase-field models for alloy solidification from a grand-potential functional. Phys. Rev. E 2011, $84,31601$.

26. Wheeler, A.A.; Boettinger, W.J.; McFadden, G.B. Phase-field model of solute trapping during solidification. Phys. Rev. E Stat. Phys. Plasmas Fluids Relat. Interdiscip. Top. 1993, 47 3, 1893-1909.

27. Bollada, P.; Jimack, P.K.; Mullis, A.M. A numerical approach to compensate for phase field interface effects in alloy solidification. Comput. Mater. Sci. 2018, 151, 338-350.

28. Folch, R.; Plapp, M. Towards a quantitative phase-field model of two-phase solidification. Phys. Rev. E 2003, 68, 010602.

29. Bollada, P.C.; Jimack, P.K.; Mullis, A.M. A new approach to multi-phase formulation for the solidification of alloys. Phys. D 2012, $241,816-829$

30. Tiaden, J.; Nestler, B.; Diepers, H.J.; Steinbach, I. The muiltiphase-field model with an integrated concept for modelling diffusion. Phys. D 1998, 115, 73-86.

31. Nestler, B.; Wheeler, A.A. A multi-phase-field model of eutectic and peritectic alloys: numerical simulation of growth structures. Phys. D 2000, 138, 114-133.

32. Bollada, P.; Jimack, P.; Mullis, A. Multiphase field modelling of alloy solidification. Comput. Mater. Sci. 2020, 171, 109085. https://doi.org/10.1016/j.commatsci.2019.109085.

33. Kundin, J.; Steinbach, I. Comparative study of different anisotropy and potential formulations of phase-field models for dendritic solidification. Comput. Mater. Sci. 2019, 170, 109197.

34. Greer, A. Phil. Trans. R. Soc. A 2003, 361, 475-495.

35. Song, Y.S.; Tourret, D.; Mota, F.L.; Pereda, J.; Billia, B.; Bergeon, N.; Trivedi, R.; Karma, A. Thermal-field effects on interface dynamics and microstructure selection during alloy directional solidification. Acta Mater. 2018, 150, 139-152.

36. Owens, R.G.; Phillips, T.N. Computational Rheology; Imperial College Press: London, UK, 2002. 
37. Bollada, P.C.; Jimack, P.K.; Mullis, A.M. Bracket formalism applied to phase field models of alloy solidification. Comp. Mat. Sci 2017, 126, 426-437.

38. Brener, E.A.; Boussinot, G. Kinetic cross coupling between nonconserved and conserved fields in phase field models. Phys. Rev. E 2012, 86, 060601.

39. Groebner, J.; Lukas, H.L.; Aldinger, F. Thermodynamic calculation of the ternary system Al-Si-C. CALPHAD 1996, 20, 247-254. 